\title{
Understanding the active site of the SARS-CoV-2 papain-like protease (PLPro) MaryAgnes Balogun ${ }^{1}$, Amy Wu Wu${ }^{2}$, Mickayla Bacorn ${ }^{3}$, Cassandra Olivas ${ }^{4}$, Christine Zardecki $^{5}$, Joseph Lubin ${ }^{6}$, Sagar Khare ${ }^{7}$, Stephen Burley ${ }^{8}$ \\ ${ }^{1}$ Morgan State University ${ }^{2}$ University of Puerto Rico, Mayagüez Campus, ${ }^{3}$ No affiliation given, ${ }^{4}$ California State University, Stanislaus, ${ }^{5}$ Rutgers Proteomics, RCSB Protein Data Bank, ${ }^{6}$ No affiliation given, ${ }^{7}$ Institute for Quantitative Biomedicine, ${ }^{8}$ RCSB Protein Data Bank, Rutgers University \\ maryagnes.balogun@yahoo.com
}

Coronaviruses are a threat to the health of the global community. Prior to the COVID-19 global pandemic caused by SARS-CoV-2, severe acute respiratory syndrome (SARS-CoV) and Middle East respiratory syndrome (MERS$\mathrm{CoV}$ ) were coronaviruses that made the species jump to infect humans. In SARS-CoV-2, papain-like proteases (PLpro) participate in cleaving the polyproteins and liberating non-structural proteins. The non-structural proteins attach to each other and create a replication transcription complex which is used for viral replication. Targeting and disrupting coronavirus proteins with drugs is one strategy for stopping future outbreaks. Studying active site residues that are highly conserved between many variants will help in developing broadly effective inhibitors. We want to be able to identify or create a drug that will work against as many coronaviruses as possible, particularly for variants that differ in the chemical character of their active sites. In order to analyze the active site of SARS-CoV-2 papain-like proteases (PLPro) we performed sequence-based comparisons of the active sites of the SARS-CoV-2 PLPro with other coronavirus PLPro enzymes using sequences obtained from NCBI, and generated computational models with Robetta. A structure-based sequence alignment was performed using the Dali server (ekhidna2.biocenter.helsinki.fi). Visualization of the molecular structures of these enzymes was performed using Mol* (RCSB.org). Preliminary results show differences in the chemical character of the active site of the SARSCoV-2 PLPro enzyme and the active sites of other experimental coronavirus PLPro enzymes. Our studies suggest that a drug effective against one PLPro enzyme may not bind to the PLPro enzyme of a different coronavirus. 